\title{
Avoiding late preterm deliveries to reduce neonatal complications: an 11-year cohort study
}

\author{
Noémie Bouchet ${ }^{1}$, Angèle Gayet-Ageron², Marina Lumbreras Areta' ${ }^{1}$, Riccardo Erennio Pfister ${ }^{3}$ \\ and Begoña Martinez de Tejada ${ }^{1 *}$
}

\begin{abstract}
Background: Late preterm (LPT) newborns, defined as those born between 34 0/7 and 36 6/7 gestational weeks, have higher short- and long-term morbidity and mortality than term infants ( $\geq 37$ weeks). A categorization to justify a non-spontaneous LPT delivery has been proposed to distinguish evidence-based from non-evidence-based criteria. This study aims to describe rates and temporal trends of non-spontaneous LPT neonates delivered according to evidence-based or non-evidence-based criteria and to evaluate the number of avoidable LPT deliveries, including severe neonatal morbidity rates and associated risk factors.

Methods: Retrospective cohort study including all LPT neonates born at a Swiss university maternity unit between January 1, 2002 and December 31, 2012. Trends of LPT neonates and neonatal complications were assessed across time using Poisson regression and risk factors for neonatal complications by logistic regression.

Results: Among 40,609 singleton live births, 4223 (10.5\%) were preterm and 2017 (4.9\%) LPT. In the latter group, 26.2\% were non-spontaneous (evidence-based: 12.0\%; non-evidence-based: 14.2\%). The most frequent indications for evidencebased non-spontaneous LPT delivery were severe preeclampsia (51.8\%) and abnormal fetal tracing (24.7\%). Indications for non-evidence-based non-spontaneous LPT deliveries were hemorrhage (36.2\%) and mild preeclampsia (15.7\%). LPT birth rates remained stable over time. The rate of neonatal complications after non-evidence-based LPT birth remained high over time (43.8\% vs. $43.5 \%$ in 2002 and 2012, respectively; $P=0.645$ ), whereas the annual proportion of neonatal complications overall showed a decreasing trend (from 38.0\% in 2002 to $33.5 \%$ in 2012; $P=0.051$ ).

Conclusions: LPT birth rates were stable over time, but neonatal complications remained high, particularly after nonevidence-indicated LPT birth. A total of 287 LPT births could have been potentially avoided if an evidence-based protocol for delivery indications had been used. Efforts should be made to avoid non-spontaneous LPT births in order to reduce neonatal complications.
\end{abstract}

Keywords: Late preterm birth, Delivery, Spontaneous, Non-spontaneous, Evidence-based, Trends, Neonatal complications

\section{Background}

Since the 1990s, the worldwide rate of preterm births (birth before 37 weeks' gestation) has been increasing and even represented up to $11 \%$ of all live births in 2010 in Europe and the USA $[1,2]$. This trend is partly correlated with the rise of medically-indicated preterm delivery in

\footnotetext{
* Correspondence: begona.martinezdetejada@hcuge.ch

${ }^{1}$ Obstetrics Unit, Department of Obstetrics and Gynecology, Geneva

University Hospitals and Faculty of Medicine, 30 Boulevard de la Cluse, 1205

Geneva, Switzerland

Full list of author information is available at the end of the article
}

order to reduce stillbirth $[3,4]$. Preterm delivery may occur as a result of spontaneous preterm labor, including the preterm premature rupture of membranes (PPROM) or medical interventions, such as labor induction or elective cesarean, that are initiated to reduce poor outcomes associated with specific maternal or fetal conditions [5-7]. Late preterm (LPT) birth, defined as delivery between $340 / 7$ and 36 6/7 weeks' gestation, represents two-thirds of all preterm births and impacts heavily on the rise of medically- indicated deliveries [8-11]. LPT neonates have long been wrongly considered as "near 
term". Beliefs about supposed "almost maturity" and the fear of stillbirth motivated weak indications for induced deliveries, such as isolated oligohydramnios or gestational hypertension [12-14].

It is now well established that LPT infants have an increased risk of morbidity and mortality compared to those born at term [15]. In the short term, they have a higher risk than term infants to suffer from respiratory distress syndrome, apnea, hypothermia, hypoglycemia, jaundice, hyperbilirubinemia, necrotizing enterocolitis and intraventricular hemorrhage [14, 16, 17]. This leads to frequent admissions to the neonatal intensive care unit (NICU) and a longer duration of hospitalization with high economic costs [18-20]. In the long term, LPT newborns appear to have an increased risk of cerebral palsy and mental retardation, as well as more behavioral abnormalities than their term peers [21, 22]. Learning disabilities and a lower socioeconomic level than their parents have also been described [23, 24]. Moreover, LPT birth and its consequences have a negative emotional and psychosocial impact on parents and families, which can last well beyond the initial period of hospitalization [25, 26].

Therefore, it is of crucial importance to determine the optimal time of delivery in order to reduce perinatal morbidity and mortality, while balancing neonatal and infant risks [27, 28]. Based on a review of the guidelines of the American College of Obstetricians and Gynecologists' [29], the obstetrics literature and published expert opinion [27], Gyamfi-Banneman et al. proposed a set of evidencebased (EB) indications to justify a LPT delivery [30, 31]. In the absence of sufficient scientific evidence, indications were considered weak to justify iatrogenic LPT delivery and were thus categorized as non-EB and included elective (non-medical) indications [13].

The aims of this study were to describe the trend of LPT births and their indications over an 11-year period according to the Gyamfi-Banneman categorization [31]. We also sought to assess neonatal complications related to LPT birth and the accompanying risk factors.

\section{Methods}

This retrospective cohort study was conducted at the maternity unit of Geneva University Hospitals, Geneva, Switzerland, and included all LPT births between January 1, 2002 and December 31, 2012. The local institutional ethics committee approved the research protocol.

\section{Study population}

The maternity unit is the largest in Switzerland with approximately 4000 deliveries per year, of which approximately $10 \%$ are preterm. We included all births of singletons between $34+0$ and $36+6$ weeks' gestation. Stillbirths and multiple gestations were excluded. We obtained the list of all newborns during the study period from the labor and delivery suite database and gathered maternal and neonatal data from the medical charts using a standardized report form. Relevant data were extracted from the following sources: the maternal and neonatal databases of the obstetrics service and the neonatal database of the pediatric department. All data were coded using a unique study number.

\section{Outcomes}

The primary outcome was the number of LPT births among spontaneous LPT, EB non-spontaneous LPT and non-EB non-spontaneous LPT deliveries. The secondary outcome was an adverse neonatal event defined by the presence of at least one of the following complications: neonatal death; NICU admission; need for ventilatory support; neonatal sepsis with bacteremia; and respiratory disease requiring oxygen or ventilatory support (composite outcome).

\section{Variables}

Data were described on an annual basis. We extracted the following maternal and obstetrical variables: maternal age; gestational age at delivery (based on the first trimester ultrasound); gravidity; parity; history and type of cesarean section; prior myomectomy; chronic hypertension; gestational hypertension; preeclampsia; cholestasis; PPROM; intrauterine growth retardation; abnormal fetal Doppler (umbilical and/or cerebral); abnormal fetal tracing; oligohydramnios; pulmonary maturation; pre-labor uterine rupture; delivery onset: spontaneous or non-spontaneous (labor induction or elective cesarean); indication for delivery; mode of delivery (vaginal delivery with or without instruments, elective or in-labor cesarean section); fetal presentation; and type of anesthesia.

Neonatal variables included gender; birth weight in grams; Apgar score at $5 \mathrm{~min}$; umbilical arterial $\mathrm{pH}$; growth retardation $(<10$ th percentile growth for gestational age); use of ventilatory support by either non-invasive ventilation (continuous positive airway pressure) or invasive ventilation (intubation and mechanical ventilation); duration of hospitalization in days; hospitalization site (maternity, $\mathrm{NICU}$ ); presence of respiratory pathologies (wet lung, respiratory distress syndrome); neonatal sepsis with positive bacteremia; neonatal malformation; chromosomal abnormalities; and neonatal death at less than 1 month.

We classified LPT birth as either spontaneous or nonspontaneous LPT. Spontaneous LPT birth was defined as the spontaneous onset of uterine contractions and cervical dilation. Women with PPROM and no other indication were included in this group. The non-spontaneous LPT birth group included women with induction of labor or elective cesarean section without contractions. This group was further subcategorized according to Gyamfi-Banneman 
et al. as either $\mathrm{EB}$ or non-EB management. Indications labeled EB were supported by strong scientific evidence based on the guidelines of the American College of Obstetricians and Gynecologists' [29], the obstetrics literature and published expert opinion [27]. EB indications for non-spontaneous LPT birth were severe preeclampsia or eclampsia, intrauterine growth retardation with abnormal testing (abnormal fetal Doppler or fetal heart tracing, oligohydramnios) or poor interval growth, acute abruption, non-reassuring fetal heart rate tracing, cholestasis, and uterine rupture prior to spontaneous initiation of labor. Indications labeled non-EB were not supported by sufficient scientific evidence and thus weaker to justify a nonspontaneous LPT delivery. Non-EB indications for LPT birth included chronic or gestational hypertension, mild preeclampsia, intrauterine growth retardation with normal testing and adequate interval growth, prior myomectomy or classic cesarean section, isolated oligohydramnios. Any other indication reviewed in medical records and not listed previously was classified as an elective delivery and categorized as non-EB LPT. These indications were in general debated within the clinical team and not supported by scientific evidence, but mainly explained by the wishes of the patient or care provider. In Gyamfi-Banneman et al., only cholestasis with bile acids $>40 \mathrm{micromol} / \mathrm{L}$ was considered as EB. Cholestasis with bile acids $<40$ micromol/L were considered as non-EB. In our study, all women with cholestasis were included in the EB group as we did not have measurements of bile acid levels.

\section{Statistical analysis}

All continuous variables were described by their mean \pm standard deviation, overall median and LPT group. Categorical variables were described by their frequencies, relative proportion overall and LPT group. A comparison of continuous variables between the three groups of LPT deliveries (i.e. between spontaneous LPT and non-spontaneous LPT or between EB and non-EB non-spontaneous LPT) was done using the Kruskal-Wallis test because of skewed distributions. Categorical variables were compared between the groups using either the Chi-square or Fisher's exact test. The overall number of complications were then stratified on the three groups of LPT and assessed across time using Poisson regression models.

Finally, we assessed the risk factors associated with the occurrence of at least one complication using a logistic regression model where the LPT group was the main predictor. We pre-specified the following variables as important risk factors for neonatal complications: mode of delivery (spontaneous, elective cesarean or cesarean during labor); pulmonary maturation (yes/no); gestational age strata ( 34 to $34 / 6$ weeks, 35 to $35 / 6$ weeks, and 36 to $36 / 6$ weeks); maternal age strata $(<20,20-29$, 30-34, 35-39 and > = 40 years); birth weight strata
( $<10$ th, 10th to 50 th, 50 to 90th, and $>90$ th percentile); and gender. Logistic regression provided maximum likelihood estimates of the odds ratios (OR) and their 95\% confidence intervals (CI). The goodness-of-fit of the model was verified using the Hosmer-Lemeshow test. The amount of variation in the likelihood of neonatal complications explained by the model was indicated by the Cox and Snell $R^{2}$. The discriminant capacity of the model was evaluated by the area under the curve assorted with its 95\% CI. Statistical significance was defined as a two-sided $P$ value of $<0.05$. Statistical analyses were performed using Stata IC 14 (STATA Corp., College Station, TX).

\section{Results}

A total of 40,609 live singleton deliveries were recorded during the 11-year study period. Among these, 4223 (10.5\%) were preterm and 2017 (4.9\%) LPT. Among the LPT deliveries, 1487 were classified as spontaneous LPT (73.7\%) and $530(26.2 \%)$ as non-spontaneous LPT. In the latter group, 243 (12.0\%) were EB and 287 (14.2\%) nonEB (Fig. 1). Maternal and obstetric characteristics are shown in Table 1. There were more women with a history of cesarean section $(P<0.001)$ and with chronic or gestational hypertension $(P<0.001$ in both $)$ in the non-spontaneous LPT than in the spontaneous LPT group. The cesarean section rate was higher in the nonEB non-spontaneous LPT (82.9\%) than in the EB group (67.1\%; $P<0.001)$. Globally, cesarean section during labor was more frequent in the non-spontaneous LPT group compared to spontaneous LPT cases (OR: 1.8; 95\% CI: $1.36-2.39 ; P<0.001)$. The proportion of each LPT subgroup remained stable over time $(P=0.665$ for spontaneous LPT; $P=0.532$ for EB non-spontaneous LPT; $P=0.609$ for non-EB non-spontaneous LPT; Fig. 2).

The most frequent indications for EB nonspontaneous LPT were severe preeclampsia (51.8\%), abnormal fetal tracing (24.7\%) and intrauterine growth retardation with abnormal testing. For non-EB nonspontaneous LPT cases, the most common indications were hemorrhage without placenta abruption (36.2\%) and non-severe preeclampsia (15.7\%). Elective indications accounted for $34.5 \%$ of non-EB non-spontaneous LPT and included various diagnoses, such as isoimmunization, maternal life-threatening conditions (i.e. breast cancer, acute renal failure, maternal sepsis), severe fetal malformation (laparoschisis, major renal defect) and maternal psychological distress related to pregnancy. Few cases were "strictly elective" without any clearly reported medical indication.

The proportion of infants hospitalized in the NICU was significantly higher in the non-spontaneous LPT compared to the spontaneous LPT group (Table 1). The number of neonatal complications in the three LPT 


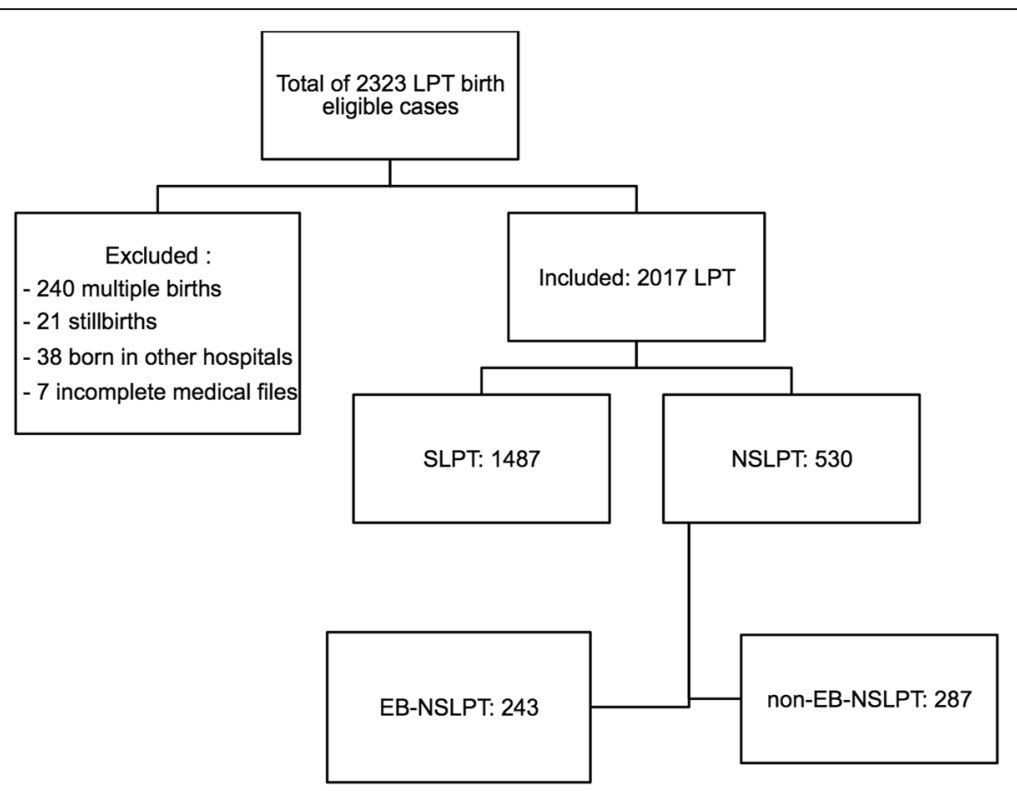

Fig. 1 Flow chart of the study population. LPT: late preterm; SLPT: spontaneous late preterm; NSLPT: non-spontaneous late preterm; EB: evidence-based

groups decreased over time, but the decrease was only statistically significant in the spontaneous LPT group $(P=0.031)$. The likelihood of neonatal complications was 3.66-fold (95\% CI: 2.75-4.87) higher in EB nonspontaneous LPT compared to spontaneous LPT, 2.04-fold higher (95\% CI: 1.58-2.63) in non-EB non-spontaneous LPT compared to spontaneous LPT, and 1.79-fold higher (95\% CI: 1.26-2.55) in EB non-spontaneous LPT compared to non-EB non-spontaneous LPT (Fig. 3). After adjustment for the main confounders (Table 2), the likelihood of complications remained significantly higher among non-EB non-spontaneous LPT compared to spontaneous LPT cases $(P<0.001)$, but there was no significant difference between non-spontaneous non-EB LPT and EB LPT $(P=0.225)$. There was a trend for higher odds of complications in the non-spontaneous EB LPT group compared to spontaneous LPT. The odds of neonatal complications were also significantly and independently increased in the in-labor cesarean section group compared to vaginal deliveries, in boys compared to girls, and in neonates with a birth weight between the 10th to 50th percentile and below the 10th percentile compared to infants with a birth weight above the 90th percentile. The multivariable model was also adjusted for maternal age and lung maturation and demonstrated a good discriminant capacity with an area under the curve of 0.83 (95\% CI: 0.81-0.85).

\section{Discussion}

Overall, approximately one-quarter of LPT deliveries at our center were iatrogenic. When stratified according to the classification proposed by Gyamfi-Banneman et al..., one-half were for non-EB indications [13]. In contrast to the current trend of increasing LPT delivery rates often attributed to non-spontaneous LPT birth, our rates remained stable over time and were slightly lower than those reported by other authors using the same criteria ( $17 \%$ by Gyamfi-Banneman et al. $[13,31]$ and $18 \%$ by Holland et al) [32]. Morais et al. reported a higher rate of $25.5 \%$ non-EB LPT, but included PPROM in this group [33]. By excluding PPROM, the rate decreased to $10.7 \%$, the lowest reported so far.

In our study, the overall rate of neonatal complications in LPT tended to decrease, although the rate after nonEB LPT birth did not change over time. As guidelines for lung maturation during the late preterm period in women at risk of delivery have changed recently, we can expect a decreasing rate of respiratory morbidity in LPT neonates in the future [34, 35]. Nevertheless, newborns born after non-EB LPT management received lung maturation more often than the other two groups and the rate of complications was higher, thus emphasizing the importance of avoiding the occurrence of these cases.

Non-spontaneous LPT newborns require more neonatal care than spontaneous LPT as they are more frequently admitted to the NICU, have a longer hospital stay and require more ventilatory support [14]. The risk persisted after adjustment for independent risk factors of neonatal complications (mode of delivery, pulmonary maturation, gestational age, maternal, birth weight and gender). By strictly avoiding non-EB LPT (14.2\% of LPT) deliveries, it might be possible to reduce the burden of neonatal morbidity up to a factor of two. We assume that potential supplementary factors may have influenced the obstetrical decision. 
Table 1 Overall maternal and obstetric characteristics and type of late preterm delivery

\begin{tabular}{|c|c|c|c|c|c|c|c|}
\hline & Overall & Spontaneous LPT $(N=1487)$ & $\begin{array}{l}\text { EB-LPT } \\
(N=243)\end{array}$ & $\begin{array}{l}\text { Non EB-LPT } \\
(N=287)\end{array}$ & $P$-value ${ }^{a}$ & $P$-value & $P$-value \\
\hline \multicolumn{8}{|l|}{ Obstetrical description } \\
\hline Maternal age: mean ( \pm SD, P50) & $31.7( \pm 5.6 ; 32)$ & $31.4( \pm 5.5 ; 32)$ & $\begin{array}{l}32.1( \pm 5.8 ; \\
32)\end{array}$ & $32.9( \pm 5.8 ; 33)$ & $<0.001$ & 0.06 & $<0.001$ \\
\hline Mean gestational age in weeks ( \pm SD, P50) & $35.3( \pm 0.8 ; 36)$ & $35.4( \pm 0.8 ; 36)$ & $\begin{array}{l}35.2( \pm 0.8 \\
35)\end{array}$ & $35.4( \pm 0.8 ; 36)$ & 0.002 & 0.005 & 0.159 \\
\hline Gravidity, n (\%) & & & & & $<0.001$ & $<0.001$ & 0.625 \\
\hline Primigravida & $714(35.4)$ & $531(35.7)$ & $110(45.3)$ & $73(25.4)$ & & & \\
\hline Multigravida & $1303(64.6)$ & $956(64.3)$ & $133(54.7)$ & $214(74.6)$ & & & \\
\hline Parity, n (\%) & & & & & $<0.001$ & $<0.001$ & 0.399 \\
\hline Primiparous & $1063(52.7)$ & $792(53.3)$ & $160(65.8)$ & $111(38.7)$ & & & \\
\hline Multiparous & $954(47.3)$ & $695(46.7)$ & $83(34.2)$ & $176(61.3)$ & & & \\
\hline Prior caesarean section, $\mathrm{n}(\%)$ & & & & & $<0.001$ & $<0.001$ & $<0.001$ \\
\hline No & $1786(88.5)$ & $1360(91.5)$ & $217(89.3)$ & $209(72.8)$ & & & \\
\hline Segmental caesarean & $213(10.6)$ & $117(7.9)$ & $23(9.5)$ & $73(25.4)$ & & & \\
\hline Non-segmental caesarean & $18(0.9)$ & $10(0.7)$ & $3(1.2)$ & $5(1.7)$ & & & \\
\hline Prior myomectomy, n (\%) & $5(0.3)$ & $2(0.1)$ & $0(0)$ & $3(1.0)$ & 0.013 & 0.254 & 0.086 \\
\hline Chronic hypertension, n (\%) & $28(1.4)$ & $7(0.5)$ & $15(6.2)$ & $6(2.1)$ & $<0.001$ & 0.016 & $<0.001$ \\
\hline Gestational hypertension, n (\%) & $63(3.1)$ & $21(1.4)$ & $24(9.9)$ & $18(6.3)$ & $<0.001$ & 0.126 & $<0.001$ \\
\hline Preeclampsia, n (\%) & $212(10.5)$ & $39(2.6)$ & $128(52.7)$ & $45(15.7)$ & $<0.001$ & $<0.001$ & $<0.001$ \\
\hline Severe preeclampsia, n (\%) & $127(60.5)$ & $0(0)$ & $127(100.0)$ & $0(0)$ & $<0.001$ & $<0.001$ & $<0.001$ \\
\hline Cholestasis, n (\%) & $3(0.2)$ & $0(0)$ & $3(1.2)$ & $0(0)$ & $<0.001$ & 0.096 & 0.004 \\
\hline Haemorrhage, n (\%) & & & & & $<0.001$ & $<0.001$ & $<0.001$ \\
\hline No & $1896(94.0)$ & $1480(99.5)$ & $233(95.9)$ & $183(63.8)$ & & & \\
\hline Placenta praevia & $37(1.8)$ & $0(0)$ & $0(0)$ & $37(12.9)$ & & & \\
\hline Acute abruption & $0(0)$ & $0(0)$ & $10(4.1)$ & $0(0)$ & & & \\
\hline Other, missing data & $74(3.7)$ & $7(0.5)$ & $0(0)$ & $67(23.3)$ & & & \\
\hline Rupture of membranes, $\mathrm{n}(\%)$ & $1090(54.0)$ & $1079(72.6)$ & $43(17.7)$ & $0(0)$ & $<0.001$ & $<0.001$ & $<0.001$ \\
\hline IUGR, n (\%) & $143(7.1)$ & $43(2.9)$ & $71(29.2)$ & $29(10.1)$ & $<0.001$ & $<0.001$ & $<0.001$ \\
\hline Abnormal doppler, n (\%) & $42(2.1)$ & $0(0)$ & $36(14.8)$ & $6(2.1)$ & $<0.001$ & $<0.001$ & $<0.001$ \\
\hline Oligohydramnios, n (\%) & $79(3.9)$ & $34(2.3)$ & $13(5.4)$ & $32(11.2)$ & $<0.001$ & 0.019 & $<0.001$ \\
\hline Abnormal fetal tracing, n (\%) & $73(3.6)$ & $0(0)$ & $73(30.0)$ & $0(0)$ & $<0.001$ & $<0.001$ & $<0.001$ \\
\hline Lung maturation, $\mathrm{n}(\%)(n=1977)$ & $373(18.5)$ & $220(14.8)$ & $48(19.8)$ & $105(36.6)$ & $<0.001$ & $<0.001$ & $<0.001$ \\
\hline Uterine rupture, n (\%) & $0(0)$ & $0(0)$ & $0(0)$ & $0(0)$ & - & - & - \\
\hline Start of delivery, n (\%) & & & & & $<0.001$ & $<0.001$ & $<0.001$ \\
\hline Spontaneous & $883(43.8)$ & $880(59.2)$ & $3(1.2)$ & $0(0)$ & & & \\
\hline Induced & $934(46.3)$ & $564(37.9)$ & $206(84.8)$ & $164(57.1)$ & & & \\
\hline Elective caesarean & $200(9.9)$ & $43(2.9)$ & $34(14.0)$ & $123(42.9)$ & & & \\
\hline Mode of delivery, n (\%) & & & & & $<0.001$ & $<0.001$ & $<0.001$ \\
\hline Vaginal & $1278(63.4)$ & $1150(77.3)$ & $79(32.5)$ & $49(17.1)$ & & & \\
\hline Elective caesarean & $200(9.9)$ & $43(2.9)$ & $34(14.0)$ & $123(42.9)$ & & & \\
\hline Cesarean in labour & $539(26.7)$ & $294(19.8)$ & $130(53.5)$ & $115(40.1)$ & & & \\
\hline Presentation, n (\%) & & & & & $<0.001$ & $<0.001$ & 0.008 \\
\hline Cephalic & $1325(89.2)$ & $983(90.7)$ & $175(93.1)$ & $167(78.0)$ & & & \\
\hline Breech & $146(9.8)$ & $92(8.5)$ & $13(6.9)$ & $41(19.2)$ & & & \\
\hline
\end{tabular}


Table 1 Overall maternal and obstetric characteristics and type of late preterm delivery (Continued)

\begin{tabular}{|c|c|c|c|c|c|c|c|}
\hline & Overall & Spontaneous LPT $(N=1487)$ & $\begin{array}{l}\text { EB-LPT } \\
(N=243)\end{array}$ & $\begin{array}{l}\text { Non EB-LPT } \\
(N=287)\end{array}$ & $P$-value & $P$-value & $P$-value ${ }^{c}$ \\
\hline Other & $15(1.0)$ & $9(0.8)$ & $0(0)$ & $6(2.8)$ & & & \\
\hline Type of anaesthesia, n (\%) & & & & & $<0.001$ & 0.111 & $<0.001$ \\
\hline Non & $347(17.2)$ & $332(22.3)$ & $9(3.7)$ & $6(2.1)$ & & & \\
\hline Epidural & $1629(80.8)$ & 1139 (76.6) & $227(93.4)$ & $263(91.6)$ & & & \\
\hline General & $41(2.0)$ & $16(1.1)$ & $7(2.9)$ & $18(6.3)$ & & & \\
\hline \multicolumn{8}{|l|}{ Neonatal description } \\
\hline Female gender, n (\%) & $928(46.0)$ & $643(43.2)$ & $127(52.3)$ & $158(55.1)$ & $<0.001$ & 0.521 & $<0.001$ \\
\hline Birth weight $(\mathrm{kg})$ : mean $( \pm S D, P 50)$ & $\begin{array}{l}2.57 \\
( \pm 0.46,2.56)\end{array}$ & $2.63( \pm 0.43,2.61)$ & $\begin{array}{l}2.19 \\
( \pm 0.47,2.14)\end{array}$ & $\begin{array}{l}2.55 \\
( \pm 0.45,2.56)\end{array}$ & $<0.001$ & $<0.001$ & $<0.001$ \\
\hline Apgar <5, n (\%) & $14(0.7)$ & $10(0.7)$ & $2(0.8)$ & $2(0.7)$ & 0.966 & 0.999 & 0.769 \\
\hline Arterial pH < 7.10, n (\%) & $67(3.5)$ & $43(3.1)$ & $15(6.4)$ & $9(3.3)$ & 0.038 & 0.140 & 0.094 \\
\hline Growth retardation <p10, n (\%) & $173(9.0)$ & $72(5.0)$ & $77(35.0)$ & $24(9.2)$ & $<0.001$ & $<0.001$ & $<0.001$ \\
\hline NICU stay, n (\%) & $828(41.0)$ & $518(34.8)$ & $161(66.3)$ & $149(51.9)$ & $<0.001$ & 0.001 & $<0.001$ \\
\hline Malformation, n (\%) & $46(5.5)$ & $24(4.6)$ & $7(4.4)$ & $15(10.0)$ & 0.033 & 0.054 & 0.138 \\
\hline Ventilatory support, n (\%) & & & & & $<0.001$ & 0.428 & $<0.001$ \\
\hline No & $1733(85.9)$ & $1322(88.9)$ & $194(79.8)$ & $217(75.6)$ & & & \\
\hline Non-invasive & $246(12.2)$ & $146(9.8)$ & $40(16.5)$ & $60(20.9)$ & & & \\
\hline Invasive & $38(1.9)$ & $19(1.3)$ & $9(3.7)$ & $10(3.5)$ & & & \\
\hline $\begin{array}{l}\text { Mean stay at NICU in days }( \pm \text { SD, P50) } \\
(n=828)\end{array}$ & $\begin{array}{l}12.1 \\
( \pm 14.2,10)\end{array}$ & $11.4( \pm 13.7,9)$ & $\begin{array}{l}13.3 \\
( \pm 13.4,11)\end{array}$ & $13.0( \pm 16.5,9)$ & 0.226 & 0.067 & 0.006 \\
\hline Neonatal sepsis with bacteremia, n (\%) & $5(0.6)$ & $2(0.4)$ & $2(1.2)$ & $1(0.7)$ & 0.458 & 0.999 & 0.367 \\
\hline Respiratory diseases, n (\%) & $419(21.1)$ & $256(17.5)$ & $72(30.6)$ & $91(32.2)$ & $<0.001$ & 0.711 & $<0.001$ \\
\hline Neonatal death, n (\%) & $4(0.2)$ & $1(0.07)$ & $2(0.8)$ & $1(0.35)$ & 0.036 & 0.596 & 0.058 \\
\hline $\begin{array}{l}\text { Presence of } \geq 1 \text { complication }{ }^{*}, \mathrm{n}(\%) \\
(n=2014)\end{array}$ & $829(41.2)$ & $518(34.9)$ & $161(66.3)$ & $150(52.3)$ & $<0.001$ & 0.001 & $<0.001$ \\
\hline
\end{tabular}

LPT late preterm, EB evidence-based, $S D$ standard deviation, IUGR intrauterine growth retardation, NICU neonatal intensive care unit, $S D$ standard deviation ${ }^{a}$ Comparisons between the three groups of LPT birth; ${ }^{b}$ comparisons between spontaneous LPT birth and EB plus non-EB-LPT birth; ${ }^{c}$ comparisons between EB and non-EB-LPT birth

Complication *: neonatal death; NICU admission; need for ventilatory support; neonatal sepsis with bacteremia; and respiratory disease requiring oxygen or ventilatory support

Elective indications accounted for $34.5 \%$ of non-EB non-spontaneous LPT deliveries and included various diagnoses, such as maternal life-threatening conditions (i.e. breast cancer, acute renal failure, maternal sepsis), fetal malformation, etc. Although these indications are not considered as EB, their severity could explain the indication for delivery and the poor neonatal outcome [36].

Antepartum hemorrhage was a frequent indication for non-EB non-spontaneous LPT birth and included placenta praevia, vasa praevia, suspicion of uterine rupture and severe genital bleeding of unknown origin. Acute abruption and uterine rupture were considered EB nonspontaneous LPT indications. Placenta praevia indicated delivery between 35 5/7 weeks and 36 6/7 weeks in 37 cases, which is in agreement with recent recommendations from the American College of Obstetricians and Gynecologists Committee on Obstetric Practice [29]. Therefore, we suggest that the categorization of Gyamfi-
Banneman et al. should include placenta praevia as an EB indication for delivery during the late preterm period, possibly after $360 / 7$ weeks if uncomplicated (no fetal growth restriction, no hemorrhage or repetitive bleeding, or no other additional EB indication). Fifteen cases were delivered due to suspected uterine rupture, although none was confirmed. By excluding the 52 cases (placenta praevia and suspicion of uterine rupture) where we disagree with the indications of Gyamfi-Banneman et al., we estimate that we could have potentially avoided 235 LPT deliveries.

The strengths of the present study are related to the use of the same categorization for LPT delivery as Gyamfi-Banneman et al., thus ensuring reproducible results and allowing to compare practices within institutions nationally and internationally. The study duration allowed to collect a large number of LPT cases (2017) with complete neonatal follow-up in a single center. 


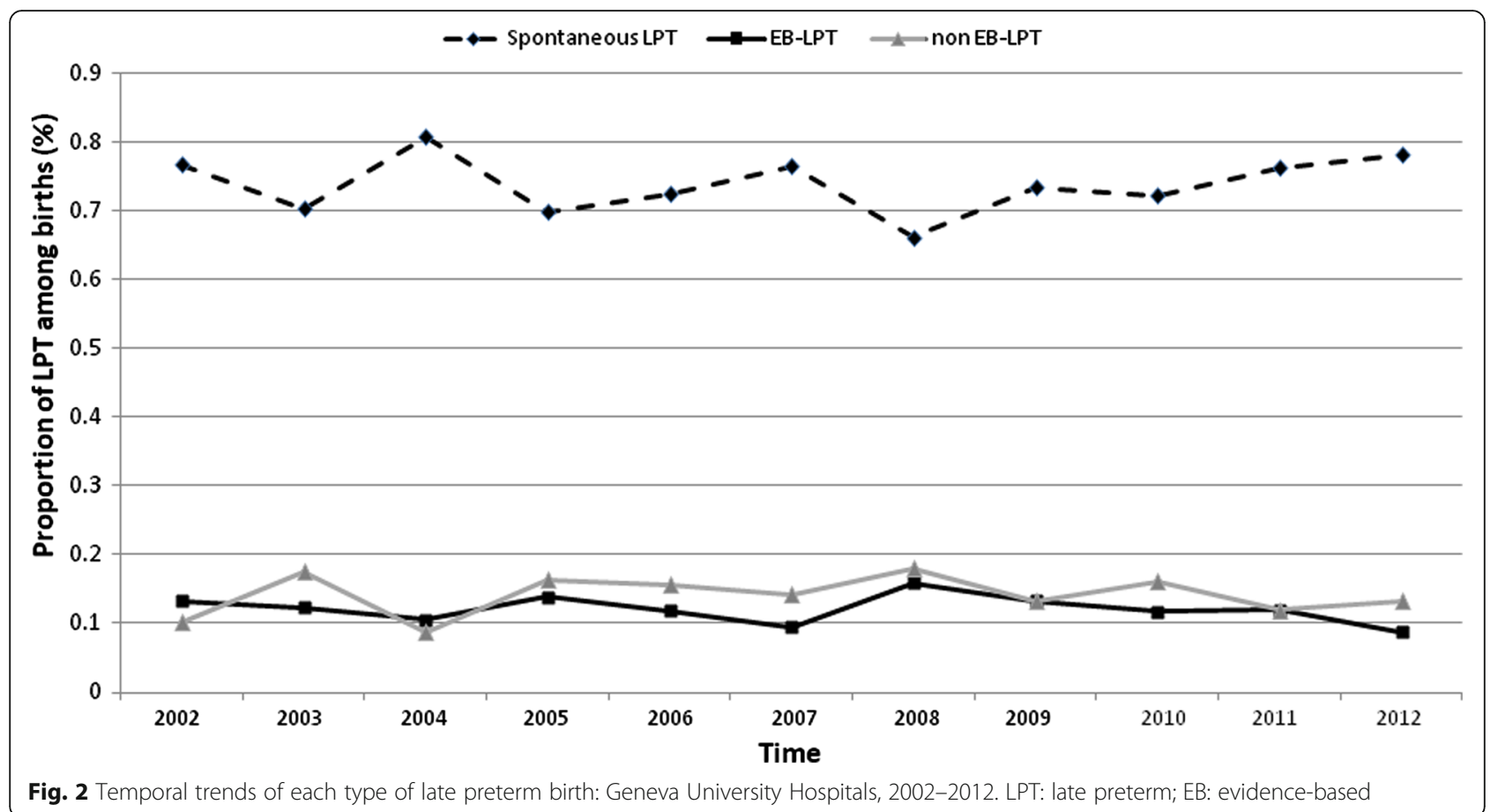

Our study has some limitations. First, its observational design means that the effect of unknown confounders could have impacted on the association between the LPT groups and neonatal complications. To account for this potential bias, we adjusted for the main known confounders. Second, we were unable to classify severe cholestasis as we did not measure bile acid and our three cases of cholestasis were classified as EB. Third, cases of
PPROM were systematically induced from 34 weeks' gestation and classified as spontaneous LPT birth. However, based on recent evidence that an expectant management of PPROM provides more benefits without increasing the rate of neonatal sepsis [37], practices related to PPROM during the late preterm period have recently changed in our maternity unit. This subgroup represented quite a large number of cases (1076) and it would now be considered

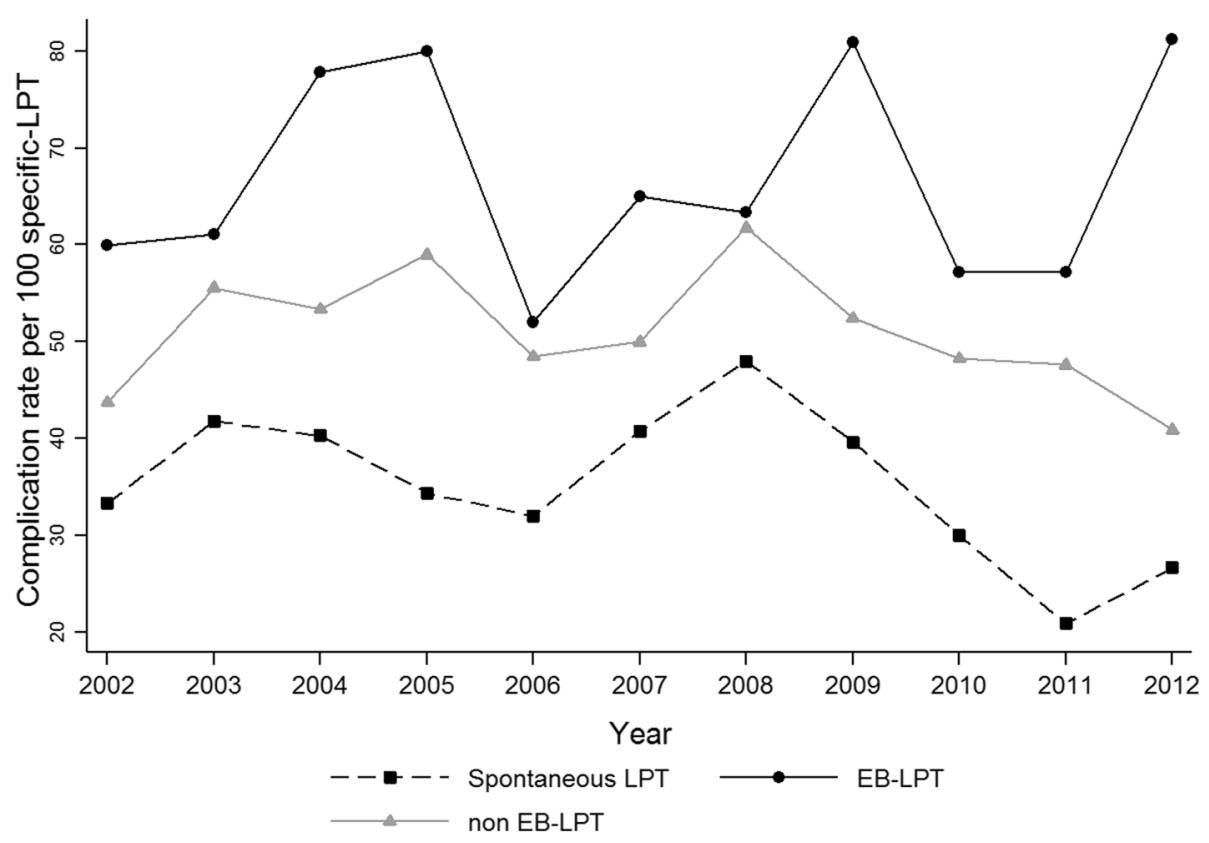

Fig. 3 Incidence rates of complications in the three late preterm birth groups. LPT: late preterm; EB: evidence-based 
Table 2 Associated factors with the likelihood of neonatal complication (multivariate analysis) ${ }^{\text {a }}$

\begin{tabular}{|c|c|c|c|}
\hline Variables & Odds ratio & $\begin{array}{l}95 \% \text { confidence } \\
\text { interval }\end{array}$ & $P$-value \\
\hline $\begin{array}{l}\text { Time periods } \\
\text { (reference 2002-2004) }\end{array}$ & & & $<0.001$ \\
\hline 2005-2006 & 0.83 & $0.59-1.15$ & 0.258 \\
\hline 2007-2008 & 0.96 & $0.68-1.36$ & 0.830 \\
\hline 2009-2012 & 0.47 & $0.34-0.64$ & $<0.001$ \\
\hline $\begin{array}{l}\text { Group of LPT } \\
\text { (reference spontaneous) }\end{array}$ & & & 0.001 \\
\hline EB-LPT & 1.49 & $0.99-2.22$ & 0.051 \\
\hline Non EB-LPT & 2.03 & $1.37-3.00$ & $<0.001^{\circ}$ \\
\hline $\begin{array}{l}\text { Mode of delivery } \\
\text { (reference spontaneous) }\end{array}$ & & & 0.0002 \\
\hline Elective caesarean & 1.53 & $0.96-2.45$ & 0.074 \\
\hline Caesarean during labour & 1.80 & $1.36-2.39$ & $<0.001$ \\
\hline $\begin{array}{l}\text { Lung maturation } \\
\text { (reference no lung maturation) }\end{array}$ & 1.01 & $0.75-1.37$ & 0.941 \\
\hline $\begin{array}{l}\text { Gestational age in weeks } \\
\text { (reference [36]) }\end{array}$ & & & $<0.001$ \\
\hline 34 & 27.3 & 19.22-38.79 & $<0.001$ \\
\hline 35 & 3.15 & $2.41-4.10$ & $<0.001$ \\
\hline $\begin{array}{l}\text { Mother's age in years } \\
\text { (reference }<20 \text { years) }\end{array}$ & & & 0.426 \\
\hline $20-30$ & 1.59 & $0.60-4.21$ & 0.350 \\
\hline $30-35$ & 1.47 & $0.56-3.90$ & 0.435 \\
\hline $35-40$ & 1.22 & $0.46-3.25$ & 0.690 \\
\hline$>=40$ & 1.61 & $0.57-4.56$ & 0.366 \\
\hline $\begin{array}{l}\text { Weight percentile categories } \\
\text { (reference }>90 \text { th percentile) }\end{array}$ & & & $<0.001$ \\
\hline 50-90th & 1.08 & $0.76-1.53$ & 0.665 \\
\hline 10-50th & 1.62 & $1.13-2.31$ & 0.009 \\
\hline 10th & 16.18 & $9.46-27.67$ & $<0.001$ \\
\hline Boy (reference girl) & 1.66 & $1.31-2.11$ & $<0.001$ \\
\hline
\end{tabular}

LPT late preterm, EB evidence-based

${ }^{a}$ Amount of variation in the likelihood of neonatal complication explained by the model was $29 \%$

PPROM without signs of complications as a non-EB indication for delivery, similar to Morais et al. [33].

Our aim was not to establish a new, strict and exhaustive classification of EB and non-EB criteria, but rather to support only EB indications. We also aimed to identify ways to reduce the number of non-spontaneous LPT deliveries in our setting and more generally. At present, this categorization is evolving and future research in the field of LPT management may help to improve the definition of $\mathrm{EB}$ and non-EB indications. In our setting, our study allowed to highlight that one-half of non-spontaneous LPT births could have been avoided if current recommendations had been applied. When possible, obstetrical settings should be encouraged to use this classification, which aims to improve the management of LPT births. A recent study reported that strengthening management policies of non-EB nonspontaneous LPT indications led to a decline in preterm birth [38]. Another study found that the presence of condition-specific obstetric protocols did not lead to detectable improvements in pregnancy outcomes [39]. Although Clark et al. reported that an association of protocols with a "hard stop" policy was the best way to improve EB medicine practices [40], the necessity for strict protocols in obstetric management remains a subject of debate among specialists.

\section{Conclusions}

The proportion of LPT births remained stable over the entire study period. One-quarter of LPT births were nonspontaneous and more than half of these were non-EB with a high risk of neonatal complications. Between 287 and 235 could have been avoided if strict criteria had been applied. Efforts should continue to reduce unnecessary LPT births in order to reduce neonatal morbidity.

\section{Abbreviations}

Cl: Confidence interval; EB: Evidence-based; IUGR: Intrauterine growth retardation; LPT: Late preterm; NICU: Neonatal intensive care unit; OR: Odds ratio; PPROM: Preterm premature rupture of membranes; SLPT: Spontaneous late preterm delivery

\section{Acknowledgements}

We thank all research midwives and administrative staff at the obstetrics service of Geneva University Hospitals for their help with database development. We sincerely thank Rosemary Sudan for editorial assistance.

\section{Funding}

The project received institutional support from the Department of Gynaecology and Obstetrics and the Department of Pediatrics, Geneva University Hospitals. There was not funding for the study.

\section{Availability of data and materials}

The datasets analyzed during the current study are available from the corresponding author on reasonable request.

\section{Authors' contributions}

NB designed the study, contributed to data collection, and drafted the initial manuscript. AGA performed the statistical analyses and interpreted the results, critically reviewed and revised the manuscript. ML contributed to data collection, reviewed and revised the manuscript. REP coordinated data collection and critically reviewed the manuscript. BMdT conceived and designed the study and the data collection, coordinated and supervised data collection, and critically reviewed the manuscript. All authors read and approved the final manuscript as submitted.

\section{Ethics approval and consent to participate}

Geneva ethics committee (Commission Cantonale d'Ethique de la Recherche) approved the protocol and the staff list.

Consent was not requested to patients as considered not necessary by the local ethics committee for the following reasons: (i) its retrospective nature, (ii) the study does not endure any risk or harm for the patient, (iii) to be able to include all cases in order to be exhaustive and avoid bias. Access to data was limited to authorized individuals to ensure patient confidentiality. Data was coded. 


\section{Competing interests}

The authors have no competing interest relevant to this article to disclose.

\section{Publisher's Note}

Springer Nature remains neutral with regard to jurisdictional claims in published maps and institutional affiliations.

\section{Author details}

'Obstetrics Unit, Department of Obstetrics and Gynecology, Geneva University Hospitals and Faculty of Medicine, 30 Boulevard de la Cluse, 1205 Geneva, Switzerland. ${ }^{2}$ Clinical Research Centre and Division of Clinical Epidemiology, Department of Community Health and Medicine, Geneva University Hospitals and Faculty of Medicine, 6 rue Gabrielle-Perret-Gentil, 1205 Geneva, Switzerland. ${ }^{3}$ Neonatology Unit, Department of Pediatrics, Geneva University Hospitals and Faculty of Medicine, 30 Boulevard de la Cluse, 1205 Geneva, Switzerland.

Received: 25 January 2017 Accepted: 28 December 2017

Published online: 08 January 2018

\section{References}

1. Zeitlin J, Szamotulska K, Drewniak N, Mohangoo AD, Chalmers J, Sakkeus L, et al. Preterm birth time trends in Europe: a study of 19 countries. BJOG. 2013;120(11):1356-65.

2. Blencowe $H$, Cousens $S$, Oestergaard MZ, Chou D, Moller AB, Narwal R, et al. National, regional, and worldwide estimates of preterm birth rates in the year 2010 with time trends since 1990 for selected countries: a systematic analysis and implications. Lancet. 2012;379(9832):2162-72.

3. MacDorman MF, Mathews TJ, Martin JA, Malloy MH. Trends and characteristics of induced labour in the United States, 1989-98. Paediatr Perinat Epidemiol. 2002:16(3):263-73.

4. Ananth $\mathrm{CV}$, Joseph KS, Oyelese Y, Demissie K, Vintzileos AM. Trends in preterm birth and perinatal mortality among singletons: United States, 1989 through 2000. Obstet Gynecol. 2005;105(5 Pt 1):1084-91.

5. Bassil KL, Yasseen AS 3rd, Walker M, Sgro MD, Shah PS, Smith GN, et al. The association between obstetrical interventions and late preterm birth. Am J Obstet Gynecol. 2014;210(6):538 e531-9.

6. lams JD, Donovan EF. Spontaneous late preterm births: what can be done to improve outcomes? Semin Perinatol. 2011;35(5):309-13.

7. McParland PC. Obstetric management of moderate and late preterm labour. Semin Fetal Neonatal Med. 2012;17(3):138-42.

8. Raju TNK. Epidemiology of late preterm (near-term) births. Clin Perinatol. 2006;33(4):751-63.

9. Hui ASY, Lao TT, Leung TY, Schaaf JM, Sahota DS. Trends in preterm birth in singleton deliveries in a Hong Kong population. Int J Gynaecol Obstet. 2014 127(3):248-53.

10. Davidoff MJ, Dias T, Damus K, Russell R, Bettegowda VR, Dolan S, et al. Changes in the gestational age distribution among U.S. singleton births: impact on rates of late preterm birth, 1992 to 2002. Semin Perinatol. 2006;30(1):8-15.

11. Lisonkova S, Hutcheon JA, Joseph KS. Temporal trends in neonatal outcomes following iatrogenic preterm delivery. BMC Pregnancy Childbirth. 2011;11:39.

12. Hankins GDV, Longo M. The role of stillbirth prevention and late preterm (near-term) births. Semin Perinatol. 2006;30(1):20-3.

13. Gyamfi-Bannerman C. Late preterm birth: management dilemmas. Obstet Gynecol Clin N Am. 2012;39(1):35-45.

14. Pike KC, Lucas JSA. Respiratory consequences of late preterm birth. Paediatr Respir Rev. 2015;16(3):182-8.

15. Boyle JD, Boyle EM. Born just a few weeks early: does it matter? Arch Dis Child Fetal Neonatal Ed. 2013:98(1):F85-8.

16. Engle WA, Tomashek KM. Wallman C: "late-preterm" infants: a population at risk. Pediatrics. 2007:120(6):1390-401.

17. Melamed N, Klinger G, Tenenbaum-Gavish K, Herscovici T, Linder N, Hod M, et al. Short-term neonatal outcome in low-risk, spontaneous, singleton, late preterm deliveries. Obstet Gynecol. 2009:114(2 Pt 1):253-60.

18. Bird TM, Bronstein JM, Hall RW, Lowery CL, Nugent R, Mays GP. Late preterm infants: birth outcomes and health care utilization in the first year. Pediatrics. 2010;126(2):e311-9.

19. Brown HK, Speechley KN, Macnab J, Natale R, Campbell MK. Neonatal morbidity associated with late preterm and early term birth: the roles of gestational age and biological determinants of preterm birth. Int Epidemiol. 2014;43(3):802-14.
20. Khan K, Petrou S, Dritsaki M, Johnson S, Manktelow B, Draper E, et al. Economic costs associated with moderate and late preterm birth: a prospective population-based study. BJOG. 2015;122(11):1495-505.

21. Teune MJ, Bakhuizen S, Gyamfi Bannerman C, Opmeer BC, van Kaam AH, van Wassenaer AG, et al. A systematic review of severe morbidity in infants born late preterm. Am J Obstet Gynecol. 2011;205(4):e371-4.

22. Saigal S, Doyle LW. An overview of mortality and sequelae of preterm birth from infancy to adulthood. Lancet. 2008;371(9608):261-9.

23. Lipkind HS, Slopen ME, Pfeiffer MR, McVeigh KH. School-age outcomes of late preterm infants in new York City. Am J Obstet Gynecol. 2012; 206(3):e221-6.

24. Heinonen K, Eriksson JG, Kajantie E, Pesonen A-K, Barker DJ, Osmond C, et al. Late-preterm birth and lifetime socioeconomic attainments: the Helsinki birth cohort study. Pediatrics. 2013;132(4):647-55.

25. McGowan JE, Alderdice FA, Boylan J, Holmes VA, Jenkins J, Craig S, et al. Neonatal intensive care and late preterm infants: health and family functioning at three years. Early Hum Dev. 2014;90(4):201-5.

26. Rogers CE, Lenze SN, Luby JL. Late preterm birth, maternal depression, and risk of preschool psychiatric disorders. J Am Acad Child Adolesc Psychiatry. 2013:52(3):309-18

27. Spong CY, Mercer BM, D'Alton M, Kilpatrick S, Blackwell S, Saade G. Timing of indicated late-preterm and early-term birth. Obstet Gynecol. 2011:118(2 Pt 1):323-33.

28. Mandujano A, Waters TP, Myers SA. The risk of fetal death: current concepts of best gestational age for delivery. Am J Obstet Gynecol. 2013;208(3):e201-8.

29. American College of Obstetricians \& Gynecologists. ACOG committee opinion no. 560: medically indicated late-preterm and early-term deliveries. Obstet Gynecol. 2013;121(4):908-10.

30. Gyamfi-Bannerman C. Obstetric decision-making and the late and moderately preterm infant. Semin Fetal Neonatal Med. 2012;17(3):132-7.

31. Gyamfi-Bannerman C, Fuchs KM, Young OM, Hoffman MK. Nonspontaneous late preterm birth: etiology and outcomes. Am J Obstet Gynecol. 2011; 205(5):e451-6.

32. Holland MG, Refuerzo JS, Ramin SM, Saade GR, Blackwell SC. Late preterm birth: how often is it avoidable? Am J Obstet Gynecol. 2009:201(4):e401-4.

33. Morais M, Mehta C, Murphy K, Shah PS, Giglia L, Smith PA, et al. How often are late preterm births the result of non-evidence based practices: analysis from a retrospective cohort study at two tertiary referral centres in a nationalised healthcare system. BJOG. 2013;120(12):1508-14.

34. Gyamfi-Bannerman C, Thom EA, Blackwell SC, Tita AT, Reddy UM, Saade GR, et al. Antenatal betamethasone for women at risk for late preterm delivery. N Engl J Med. 2016;374(14):1311-20.

35. Society for Maternal-Fetal Medicine Publications Committee. Implementation of the use of antenatal corticosteroids in the late preterm birth period in women at risk for preterm delivery. Am J Obstet Gynecol. 2016:215(2):B13-5.

36. Kim SA, Lee SM, Kim BJ, Park CW, Park JS, Jun JK, Yoon BH. The risk of neonatal respiratory morbidity according to the etiology of late preterm delivery. J Perinat Med. 2017:45(1):129-134.1.

37. Morris JM, Roberts CL, Bowen JR, Patterson JA, Bond DM, Algert CS, et al. Immediate delivery compared with expectant management after preterm pre-labour rupture of the membranes close to term (PPROMT trial): a randomised controlled trial. Lancet. 2016;387(10017):444-52.

38. Gyamfi-Bannerman C, Ananth CV. Trends in spontaneous and indicated preterm delivery among singleton gestations in the United States, 20052012. Obstet Gynecol. 2014;124(6):1069-74.

39. Bailit JL, Grobman WA, McGee P, Reddy UM, Wapner RJ, Varner MW, et al. Does the presence of a condition-specific obstetric protocol lead to detectable improvements in pregnancy outcomes? Am J Obstet Gynecol. 2015;213(1):e81-6.

40. Clark SL, Frye DR, Meyers JA, Belfort MA, Dildy GA, Kofford S, et al. Reduction in elective delivery at $<39$ weeks of gestation: comparative effectiveness of 3 approaches to change and the impact on neonatal intensive care admission and stillbirth. Am J Obstet Gynecol. 2010;203(5):e441-6. 